\title{
An Investigation into the Role of Mobile Technology in Collaborative Disease Management for Persons Living with Type 2 Diabetes
}

\author{
S. Sultan ${ }^{1, *}$ and P. Mohan ${ }^{1}$ \\ ${ }^{1}$ The University of the West Indies, St. Augustine, Trinidad and Tobago
}

\section{Abstract}

Diabetes and other chronic non-communicable diseases represent a leading threat to health care and human development. The authors discusses how mobile technology can achieve collaborative disease management (CDM) within a population living with Type 2 diabetes. We introduces a framework for CDM using mobile technologies. While it can be applied to the management of any disease, we describe a specific instance for diabetes peer-support. In this paper, we validate the framework through the development and testing of a Mobile Health initiative called Mobile Diabetes Self-Management Support (DSMS). It allows persons living with diabetes to learn from each others' experiences through a virtual support group. The paper presents two user studies conducted. The first study captured the participants' initial perceptions of the application in a controlled environment and the second study investigated system usage and collaboration when the application was used in their personal settings.

Keywords: CNCDs, collaborative disease management, diabetes self-management support, mobile health, peer-support, remote self-management, social-networking, telehealth.

Received on 15 September 2014, accepted on 20 February 2015, published on 18 May 2015

Copyright (C) 2015 S. Sultan and P. Mohan, licensed to ICST. This is an open access article distributed under the terms of the Creative Commons Attribution licence (http://creativecommons.org/licenses/by/3.0/), which permits unlimited use, distribution and reproduction in any medium so long as the original work is properly cited.

doi: 10.4108/phat.1.1.e6

\section{Introduction}

We are quickly approaching the Millennium Development Goals (MDG) deadline and much work still has to be achieved in the area of Information and Communications Technologies (ICTs). More than one-third of the MDG goals are health related. This paper addresses these areas by presenting a framework which harnesses the efficiency and effectiveness of a mobile technology platform with a greater reach. The MDG focuses on the provision of basic social services such as health care, nutrition, education, health care etc. Existing research has shown how ICT can be used as a means of achieving these goals but not much research has been done on the use of technologies in areas where ICT is substandard. This paper explores how mobile technologies

${ }^{*}$ Corresponding author. Email: ssultan@ gmail.com can be used for collaborative disease management. It presents a case for the management of a chronic-noncommunicable disease called diabetes but the framework can be extended to other diseases.

In Trinidad and Tobago, the major causes of deaths are attributable to chronic non-communicable diseases (CNCDs). These are cardiovascular disease (25.1\%), diabetes $(13 \%)$, cancer $(13 \%)$ and cerebro-vascular disease $(10.4 \%)$, totaling $61.5 \%$. Cardiovascular disease and diabetes, in particular, are major CNCDs in the Caribbean and other regions of the world.

Diabetes mellitus or simply diabetes is a CNCD whereby a person does not produce enough insulin or the body cannot respond properly to the insulin that is produced resulting in a high blood sugar (glucose) level. Diabetes that is not properly managed can lead to major complications including kidney failure, heart disease, retinopathy and neuropathies. 
Many of these problems can be prevented but current health care practices do not embody the necessary mechanisms to initiate change [1].

The highest density of persons living with diabetes is found in the Caribbean and in the Middle East, with their population numbering from twelve to twenty percent of their total populace [2]. At the individual level, in many instances, the costs associated with treating the disease can very costly, severely limiting family members' prospects for the future [3].

In 2012, the International Diabetes Federation reported that 38.4 million people in North America and the Caribbean (NAC) region have diabetes and by 2030 this number will rise to 51.2 million. This figure accounts for $11 \%$ of adults having the disease. Diabetes can lead to serious and costly complications estimating 199.4 billion Euros spent on treating diabetes in the region.

The annual number of deaths in 2012 caused by diabetes mellitus in NAC was estimated at 287, 020. Globally this figure amounted to 4.8 million. These figures are moderate given the fact that many of the deaths unclassified are caused by complications related to the disease.

It should be noted that diabetes and other CNCDs are lifestyle diseases and control can be achieved through behaviour modification. A preventative approach for persons suffering with CNCDs is to take better care of themselves causing them to lead the lives they have reason to value. This will ultimately result in more productive lives and less impact on the economy. Clearly then, tackling diabetes and other CNCDs through behaviour modification can play a significant role in developing countries.

Diabetes, like other CNCDs, requires lifelong, continuous medical care. However, health care services in the Caribbean and other parts of the world are oriented to acute medical care. These problems are episodic in nature and usually require urgent diagnosis, testing and relief of symptoms associated with the disease. Preventative care, on the other hand, requires a different model of which current health care systems fall short [1].

Diabetes Self-Management Support (DSMS) emphasizes that without sustained support, diabetics will not succeed in the self-management process. In the Caribbean, existing DSMS initiatives are not easily accessible to the larger diabetic population and are not as effective in the long term because they lack regular follow up and feedback [5]. Furthermore, they are not tailored to the specific needs of the patient and change management techniques are required to ensure the DSMS practices are not only adopted but sustained [6]. For doctors and other health caregivers to provide this support on a one-on-one or ongoing basis is often too costly and time consuming [7]. Therefore a more interactive and personalized approach is necessary at the primary health care level with patients, their families and other health care providers.

Advances in information and communications technology have introduced new approaches that support health care delivery. These advances fall under the telehealth research branch and enable support of patients as active consumers in a health care delivery system that is evolving from an institution-centric to a patient-centric model. This research is motivated by the patient-centric model and proposes a framework for collaborative disease management using mobile technologies. The objective of the framework is to allow users with similar disease management interests to virtually gather and share experiences, ask questions and provide support and problem-solve remotely through the use of mobile devices.

Section 2 of the paper defines the term collaborative disease management and explains the role peer support plays. Section 3 highlights the existing work done in the area of peer support using telehealth. Section 4 introduces the framework surrounding the use of mobile technologies for collaborative disease management. Section 5 describes the design and development of a collaborative disease management system that uses mobile telechnology, which represents an instantiation of the framework. Sections 6 and 7 present two user studies: one in a controlled environment and the other, in the real world. Section 8 discusses the results and how the design and development of the system is used to validate the framework and lays the foundation for future work.

\section{Collaborative Disease Management and Peer Support}

Collaborative Disease Management (CDM) refers to interventions that improve outcomes of disease that have been developed on the basis of social learning and self-regulation theories [8]. Through the use of CDM, patients mitigate the complications associated with the disease by learning from others in their community and practicing better self-management behaviours. This reduces both the individual and national costs of managing the disease e.g. medication, clinic visits, surgeries etc. CDM lessons the burden that is placed on the limited health care resources of a nation. Instead of every patient competing for the same fixed set of limited resources, CDM leverages the power of the individual experiences that come from living with a disease. Therefore one of the equally important aspects of CDM is that it has the power to be self-sustainable since it utilizes the human resources of the same people that live with the disease.

Peer Support is one form of CDM. It is based on the idea that patients, although they have problems, do have some resources that can be leveraged to help other patients (Holthe, 2006). It is fuelled by the fact that peers have a common interest and therefore peer support takes place between people who are in equal positions - the principle of reciprocity. Peers are people in similar life situations. Therefore both people with the illness and their families can benefit from support. A peer worker is a person with a reasonably longer exposure to the illness and experience in coping with the illness and related problems. The peer worker's matured experiences are recognized as resources to be utilized. 


\subsection{Peer-Support Models}

Heisler (2006) has shown that models that build on peer support have proven to be both successful and cost effective as they combine the traditional peer support, that is, encouragement from someone else with a similar condition, along with a more structured education programme and assistance. Research has shown that peer support provides informational support, emotional support and mutual reciprocity which leads to an increased confidence, increased understanding of self-care and a perceived feeling of community. This translates to better lifestyle practices, prevention of further complications and an overall improved quality of life.

Presently there exist several models of peer support falling into two main categories: face-to-face selfmanagement programs and remote self-management programs. In the first category there are professional-led groups, peer-led groups, peer coaches and community health workers. In the second category we have telephone-based support and web/email-based programs. The research described in this paper intends to extend the remote model to include mobile devices such as mobile phones and physiological meters for home use. Table 1 compares existing peer support models on the following features: delivery - indicates if the support is provided face-to-face or remotely; coordinator - identifies who provides the support; communication - indicates if the support is provided in a group, on an individual basis or both; identity - states whether or not the identity of the participant is known or not; flexibility - a measure of how easy it is to coordinate the initiative in terms of availability of participants and their physical locations

\section{Table 1. This Peer-Support Models}

\begin{tabular}{|c|c|c|c|c|c|}
\hline \multirow[t]{2}{*}{ Type } & \multicolumn{5}{|c|}{ Features } \\
\hline & $\begin{array}{l}\text { Delive } \\
\text { ry }\end{array}$ & $\begin{array}{l}\text { Coordinato } \\
\mathbf{r}\end{array}$ & $\begin{array}{l}\text { Communic } \\
\text { ation }\end{array}$ & $\begin{array}{l}\text { Identit } \\
\text { y }\end{array}$ & $\begin{array}{l}\text { Flexibil } \\
\text { ity }\end{array}$ \\
\hline $\begin{array}{l}\text { Professio } \\
\text { nal-led }\end{array}$ & $\begin{array}{l}\text { Face- } \\
\text { to-face }\end{array}$ & $\begin{array}{l}\text { Health care } \\
\text { provider/edu } \\
\text { cator }\end{array}$ & Group & Known & Low \\
\hline Peer-led & $\begin{array}{l}\text { Face- } \\
\text { to-face }\end{array}$ & $\begin{array}{l}\text { Person living } \\
\text { with illness }\end{array}$ & Group & Known & Low \\
\hline $\begin{array}{l}\text { Peer } \\
\text { Coaches }\end{array}$ & $\begin{array}{l}\text { Face- } \\
\text { to-face }\end{array}$ & $\begin{array}{l}\text { Survivor of } \\
\text { illness }\end{array}$ & Individual & Known & Medium \\
\hline $\begin{array}{l}\text { Health } \\
\text { Workers }\end{array}$ & $\begin{array}{l}\text { Face- } \\
\text { to-face }\end{array}$ & $\begin{array}{l}\text { Figure head } \\
\text { in } \\
\text { community }\end{array}$ & Both & Known & Medium \\
\hline Landline & Remote & $\begin{array}{l}\text { Any of the } \\
\text { previously } \\
\text { mentioned }\end{array}$ & Both & $\begin{array}{l}\text { Anonym } \\
\text { ous }\end{array}$ & High \\
\hline
\end{tabular}

\begin{tabular}{|c|c|c|c|c|c|}
\hline $\begin{array}{l}\text { Web \& } \\
\text { Email }\end{array}$ & Remote & $\begin{array}{l}\text { Any of the } \\
\text { previously } \\
\text { mentioned }\end{array}$ & Both & $\begin{array}{l}\text { Anonym } \\
\text { ous }\end{array}$ & High \\
\hline
\end{tabular}

\section{Existing Work}

Telecommunications technology can contribute significantly to the organizing of peer networks remotely, especially in low frequent patient units living dispersed over wide geographical areas [9]. Furthermore, it can fill the gap in low resource settings where traditional peer support is either not available or not easily accessible. This section describes some of the existing work done in technology-facilitated peer support.

PatientsLikeMe is a website that allows patients to find other people with a similar condition to learn how they treat their disease [10]. The patient enters personal health data and this information is used to generate a listing of possible candidates to connect and share with. Each user in the system is represented by an image called a nugget which highlights the part of the body that is affected by the illness, how long the person is living with the disease, and other related health information.

Curetogther is another web-based system that allows the patient to search for resources by condition, symptom or treatment [11]. The patient is also provided with interface to complete surveys based on his condition, to enter health measures that the patient wishes to track (including weight, caloric intake, sleep, exercise etc.) and connect with other patients.

This research focuses on providing remote peer-support to a population of 45-75 year olds. In terms of designing interfaces for older populations, $[12,13]$ present the findings from designing a web-based personal health applications for older adults and provides some design considerations to consider when developing applications for this target population.

Recent research has also been conducted to investigate how mobile applications can assist individuals in their daily health regimes. Connelly et. al (2006) examine how mobile applications can enhance self-monitoring by providing real-time feedback and employing persuasive technology. Patrick et al. (2009), explores the effects on a group of patients receiving support for their weight management via SMS. The results showed that participants would recommend this type of intervention for weight control to friends and family.

In a recent study [16], the authors investigate the use of social networking in the FewTouch diabetes mobile application [17]. The users primarily used the social aspect of the application for arranging meetings and sharing recipes. Preliminary results show that the social interaction by regular face-to-face meetings and online interaction can influence self-management positively.

In summary, most of the existing peer support systems are web based and not everyone has access to computers or a reliable Internet connection, especially in developing countries where chronic non-communicable diseases are 
more prevalent. Furthermore, there is limited research on the use of mobile phones for CDM using peer-support in patient populations. Another notable feature is that group identification and formation is usually on the part of the user. If the population is large this can be an extensive search. Therefore, there is a need to offer a more easily accessible and readily available channel for people to engage in peer support remotely. Lastly, there are no existing protocols and standards for sharing health data among different mobile devices.

\section{The Framework}

Mobile Health is a rapidly growing area of research. It can be defined as the use of mobile technologies for the provision of health care services. The use of mobile phones, in particular, for the delivery of health care is becoming more widespread especially in developing countries where access to health care resources is limited but mobile phone usage is high. Existing mobile health projects have focused on communication structures that connect the patient to his/her health care provider. ICT in this context is intended to make the delivery or exchange of information between these entities more efficient. The research presented in this paper, however, focuses on engaging the communication between patient and patient, built on the social networking culture of the power of communities. In the proceeding scenario, we present a use case scenario for CDM using mobile technologies, and then we discuss the different components of the supporting framework.

Meet Rajesh the farmer. Rajesh has been living with diabetes for the last 15 years. He has recently started noticing that his vision is becoming impaired on occasion. Rajesh suspects it may be related to spikes in his sugar level but he does not have any written record of his past blood sugar readings to confirm this. He lives in a rural village and is not scheduled to visit the clinic for another three months. He knows that one of his neighbours, Carlos, also lives with diabetes but he is not sure when next he will be meeting him. Rajesh wants to take action soon before his condition starts to worsen. This is where CDM can make a difference.
The objective of the framework is to demonstrate how CDM using mobile technologies allow users with similar disease management interests to virtually gather and share experiences, ask questions and provide support and problem-solve remotely through the use of mobile devices. The term problem-solve in this context refers to the person's ability to identify patterns in their disease management routine and how this information can be used to influence health outcomes.

The framework, as shown in Figure 1, is broken up into five components: the CDM Mobile User, The Support Context, Data Management and Sharing, Socio-Economic Aspect: Facilitators \& Inhibitors and Security \& Privacy.

\subsection{The CDM Mobile User}

CDM mobile users share the characteristics of mobile users, self-managed people and collaborative people.

Mobile users are seen as always on the go, heavily influenced by their environment, are constrained by the affordances of their mobile devices and have the feeling of always being connected. Self-managed peoples' main objective is to better manage their illness. They see prevention as the key to control. Collaborative people are willing to share and see the exchange of knowledge as a valued resource. Given these general characteristics, special consideration must also be given depending on the user demographics of the particular target group.

\subsection{The Support Context}

For any collaboration to take place, there is a need to establish a community with defined roles and privileges within a shared context. Thus, mechanisms to discover other users and connect with them and their interests in a mobile environment and in a seamless manner are necessary. A key issue for coordination among users is the implementation of a group formation using patient specific data and constantly changing individual health goals. 


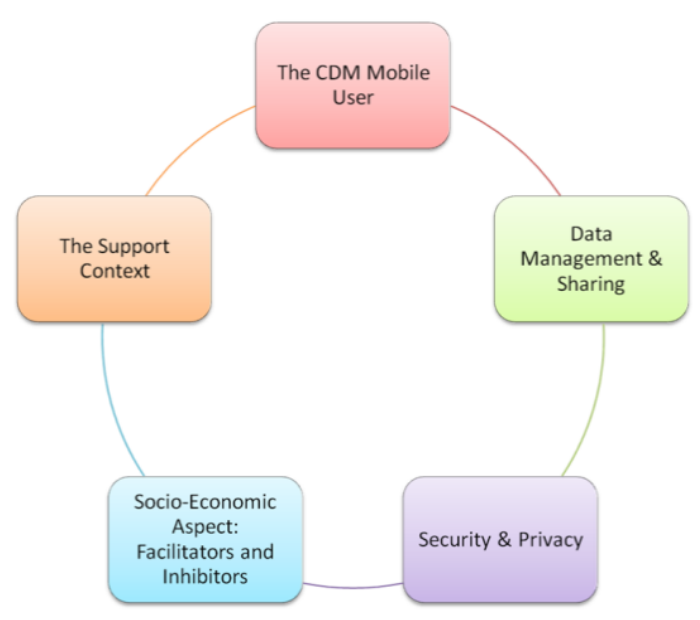

Figure 1. Collaborative Disease Management Using Mobile Technologies Framework

\subsection{Data Management and Sharing}

Existing CDM interactions typically proceed in a web-based fashion. With a mobile-based system, new protocols for data sharing are needed. New constructs are required to enable personalized sharing options that offer control over data visibility and exchange in a mobile context.

\subsection{Socio-Economic Aspect: Facilitators and Inhibitors}

The success of collaborative systems depends on the socio-economic incentive mechanisms that enable each user to work for the "common good". Challenges such as lack of motivation where some users may choose not to participate or may participate infrequently may occur; especially since users are not located in the same physical setting. Therefore there is a need for an appropriate incentive system in place, with some form of reward, in order for CDM to occur else the intended support will be ineffective.

\subsection{Security and Privacy}

A system that allows users to interact directly has major security and privacy implications. Patients (and by extension their devices) may have different roles, privileges and security settings. In the case of sharing in a CDM context, the health data stored is of a sensitive nature. Therefore users should control who accesses data stored on them.

In the next Section we demonstrate how the framework was realized through the design and development of a collaborative disease management system called Mobile DSMS. 


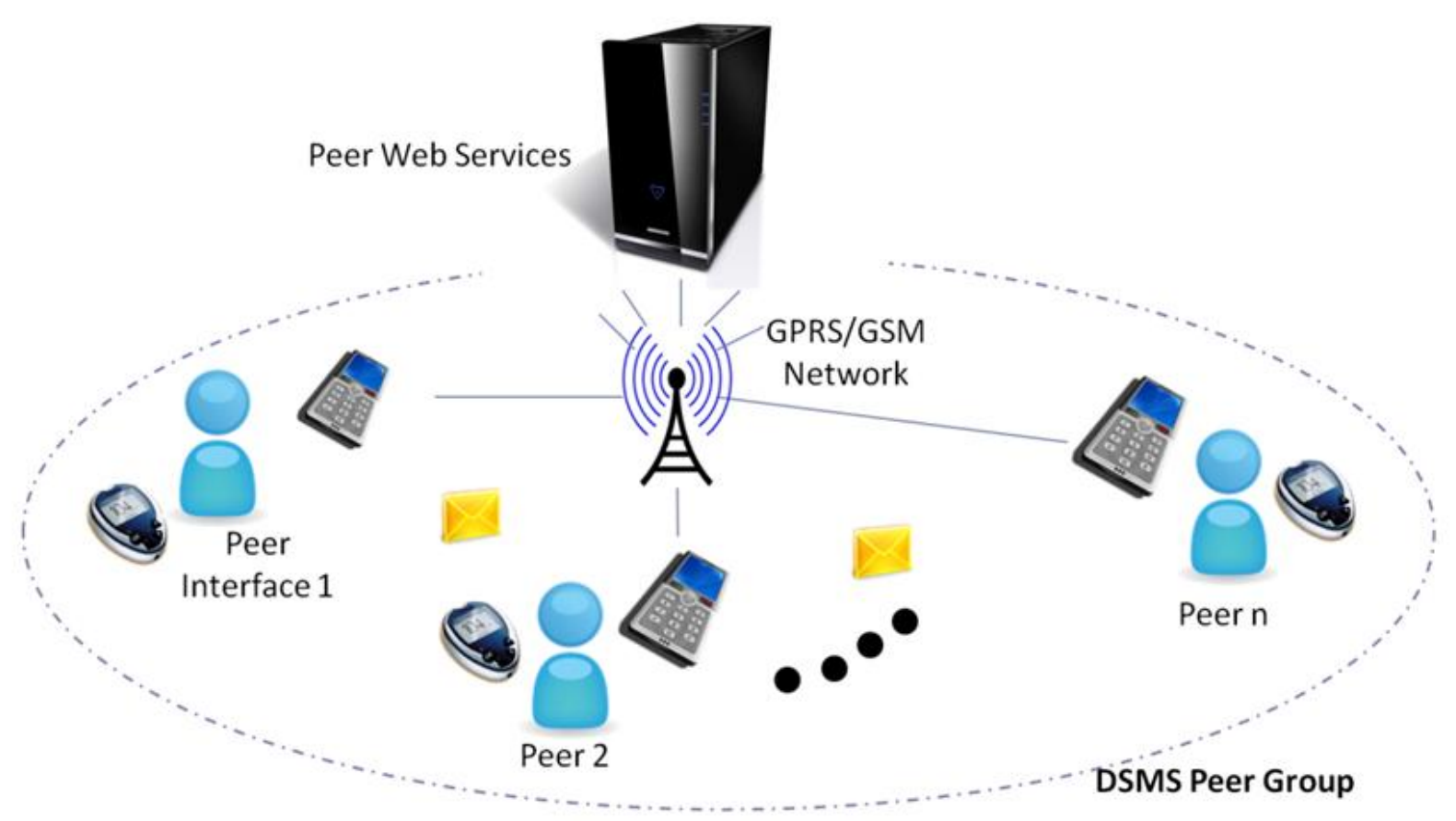

Figure 2. The Mobile DSMS System Architecture

\section{The Mobile Diabetes Self-Management Support System (Mobile DSMS)}

Our research validates the framework presented in Section 4 through the design and development of a collaborative disease management system called Mobile DSMS that provides DSMS using mobile telephony. The system allows persons living with diabetes to use a mobile phone to record observations of their daily life (ODLs) and share their knowledge and experience of their disease with their peer group. In this context, ODLs include the patient's blood sugar readings, exercise in minutes, caloric intake and current location. The assumption is that through regular monitoring of one's health and learning from others who share similar experiences, the patient's overall health status will improve thereby decreasing the mortality rates associated with poor self-care practices. Users will now be empowered to manage their own health through the learning and sharing processes.

The Mobile DSMS system is made up of three main parts: the peer interface, the peer web services and the communication network as shown in Figure 2. The peer interface includes the person living with the disease, also referred to as the patient, the patient monitoring equipment and the application running on the patient's mobile phone. The patient monitoring equipment consists of the patient's blood sugar meter - the device used to measure the patient's blood sugar level. In the future, when the system is scaled to include the tracking of other diseases, the respective sensor/meter will be included. The mobile application is the main interface with the diabetic patient. The application runs on a mobile Smartphone running an Android OS Jellybean 4.1 with a screen size of 3.5 inches. The application also 
uses the HTML5 standard for the development of the application's interface including storage of all the ODL data entered by the user. Storing the patients' data securely on the phone ensures that patients will own their personal data and have the authority and freedom to share data with whomever they choose. A 4 Gigabyte micro SD storage card is used to hold all the extra resources needed by the application so as to expand the storage capability of the phone.

The peer web services comprise a remote server hosting a variety of web services used to coordinate the sharing activities of each patient using the Mobile DSMS system. The services allow the users to share information relating to the ODLs and other health related information. The peers control what other peers within their group can see regarding their personal health data. Peers can also share experiences within the wider Mobile DSMS patient population through a forum-like feature. The features of the system are described further in Section 5.2.

The mobile phones communicate with the server securely using GPRS over a GSM network. A notification system is used to distribute alerts to all members of the Mobile DSMS community. All patient-specific information to be transferred is encrypted. The type of communication protocol used depends on the nature of the data, the sensitivity of the data being transferred and the necessity to ensure that the data arrives at the intended destination. For example, notifications are used to send out reminders of scheduled group meetings or when a peer receives a new message.

\subsection{User Characteristics}

The pilot system is first targeted to persons living with Type 2 diabetes in Trinidad and Tobago. The target users are between the ages of 45-75 years of age and already enrolled in some form of self-management program; this is necessary to separate and measure the effect of the mobile technology. The CDM mobile user essentially represents a peer in the system. A peer can either be a person living with diabetes or a caregiver to a person living with diabetes. Each peer is automatically assigned to a group based on a custom group formation algorithm. As mentioned before, peers own their data and have control over who can view data stored on them and what type of data they wish to share. Peers can remain anonymous within their group through the use of an alias or nickname.

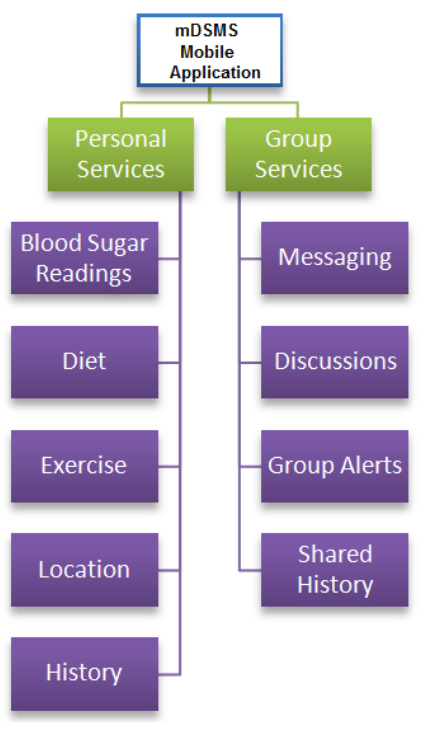

Figure 3. Peer's Mobile Interface Functional Design

\subsection{System Features}

Figure 3 shows how the peer's mobile interface is logically broken up into two parts: personal services and group services. The personal services component provides features that enable the patient to monitor and record information relating to their current health care behaviours. The main behaviours being recorded and monitored include: blood glucose readings, blood pressure readings, exercise performed and carbohydrate intake. Research has shown that these areas have a strong relationship with the patient's self-management practice [1]. These processes of recording and monitoring are essential to the decision making process. The patient is also able to review a history of past ODLs. This process of refection helps the patient to have a clearer picture of his/her current health care status, identify patterns of behaviour and problem-solve. For example, if Rajesh is experiencing impaired vision occasionally, he can review 
his past readings, nutritional intake and physical activity to see if there is a trend. Once the patient is equipped with the necessary tools and data he can then make more informed decisions regarding his health care.

The group services component provides features to allow peers to connect and learn from each other by sharing information relating to their health care behaviours. These include a messaging feature, a discussions feature, a trivia feature and an alerts feature. The message feature allows the peer to send messages to other members in the group. In the case of Rajesh, the farmer, he can send a message via his mobile phone to his friend Carlos and Carlos can help him troubleshoot based on his history of ODLs. The discussions feature allows patients to share information with all the peers that belong to their group. Patients can post questions and discuss relevant issues relating to self-care management. The trivia feature allows the peer to test their individual knowledge on diabetes self-management education. Lastly, the notifications feature generates reminders for group meetings and provides information on local health care community events.

As part of the group membership, peers share their daily ODLs with the other members of the group. This is viewed through the group's results (shared) interface. Here the peer is provided with a view of his ODLs along with the ODLs of his group members. Screenshots of the mobile application are presented in Figure 4.

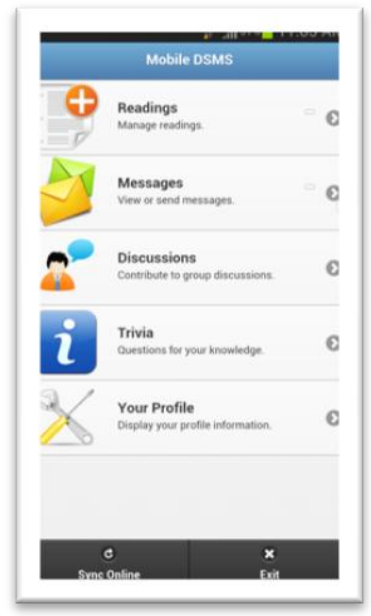

(a)

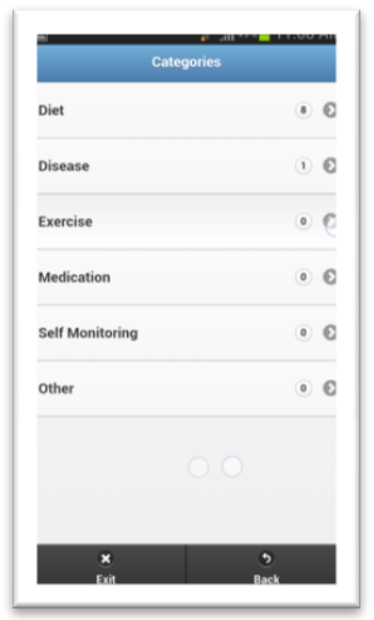

(b)

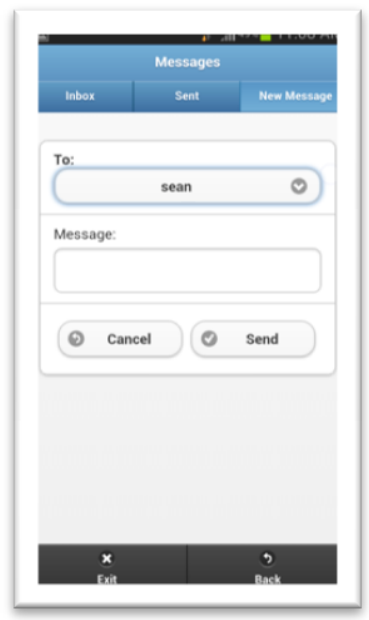

(c)

Figure 4. Mobile Application Screenshots: (a) Main Menu (b) Discussion Categories (c) Send Message to Group Member Interface

\subsection{External Interfaces}

The main source of input is from the peer and the peer's health care meter. The blood sugar reading is taken from a glucometer in $\mathrm{mg} / \mathrm{dl}$. The daily exercise is measured as the amount of time in minutes the patient exercised for the day. The patient selects the level of carbohydrates contained in their last meal(s). This information is shared within the group. Members can also share information in the form of messages within the group, discussion topics posted and replies to posts. Output from the system will be displayed on the mobile application screen and a subset of data will be stored in a database located on the phone to allow for offline access.

\subsection{Constraints}

During the development of the mobile application, the designer took into account the hardware constraints of the phone. In particular, the small screen size, limited input modes, the slower processing capabilities and the limited storage space. Given the demographic of the target group, one of the main challenges when designing an application on smaller devices such as the Smartphone, is that the user may have vision impairment. Therefore other forms of input and output are exploited including guided voicebased instructions through a text-to-speech module and voice input via a speech-to-text module. A magnified onscreen keyboard was implemented to allow the user to enter values more freely and a memory card was used to expand the storage capacity of the phone.

\section{User Study 1 - High Fidelity Prototype}

The two studies reported in this paper are part of a larger project looking at how to improve the self-care practices 
of an elderly, chronically ill patient population. In particular we are working with patients who have Type 2 diabetes. We obtained approval for these studies from the Ethics Committee at the University of the West Indies, St. Augustine campus.

By developing the DSMS mobile application, we hope to assist these patients by providing a platform for patients to connect and share with each other to better understand the disease itself as well as how lifestyle changes can improve their health status. Presently there is no application that is suitable for this target population, which has the added constraint of a high portion of users with vision impairment.

Since this type of application was new to the target population and most of the participants used their phones primarily for making phone calls, it was necessary to meet with the users from the initial design stages of the application to identify what type of user interface was suitable and special design considerations that should be noted.

In a previous study [18], conducted in January 2012, paper prototypes were developed and a focus group was conducted to gauge feedback of the proposed design for the mobile application interface. The outcomes from this study were used to refine the interface's design and a high-fidelity prototype was developed based on this design. This new prototype was developed to run on a Smartphone device.

The aim of the first study presented in this section is to evaluate the usability of the application running on the Smartphone and to investigate the patients' perceptions of the mobile application's design, functionality and its navigation structure. Specifically the research questions were:

- Will the patient be willing to use an application like this one?

- What does the patient like most about the application?

- What does the patient like least about the application?

- What changes are necessary?

- What challenges are observed when the patient performs defined tasks using the application?

The study took place at a health centre in a rural village in South Trinidad. The participants voluntarily participated and were practising some form of self-management. A total of twenty participants were recruited consisting of thirteen women and seven men. All were Indo-Trinidadian (of Indian ancestry). The average age of the participants were 65.8 years (S.D. 7.6 years). In terms of the familiarity with mobile phone, all participants owned a cell phone and the majority used it primarily for making calls.

After obtaining informed consent from the participants, we presented an overview of the project and the purpose and procedure of the study. The study started off with a demonstration of the application's features so that participants will become familiar with its functionality. This was followed by task-based interactions with the prototype. At the end of the task-based interactions, each participant filled out a questionnaire focused on their impressions of the system. The entire study lasted 2 hours.

The main findings were that $75 \%$ of the participants said that they would use the application on a regular basis. The remaining $25 \%$ were not sure if they would use it. The majority found it easy to understand and admitted that more time was needed to get comfortable with navigating through the different features. The most popular reasons for liking the application were: it is simple, it is very educational, patients like the concept of sharing and connecting with other people, patients like using the 'fancy' phone, and having easier access to important information relating to the disease. In terms of which features they did not like, $10 \%$ found the application difficult to understand and had trouble reading the screen. It was later explained that these participants did not have their reading spectacles with them that day. This is where the text-to-speech module helped. The participants were informed that a caregiver could also be used to assist them with the use of the application. Some of the changes that were suggested included: being able to use the system on their personal phone (versus the phone provided), having more time to use the application and having more assistance when using the application.

During the interaction sessions, participants were encouraged to think-out-loud so as to better record their perceptions while using the application. Many of the participants were not familiar with a touch screen phone but found it easier to manage over their personal keyboard-based phones. Some participants caught on very quickly with the tasks while others needed more assistance/reminders. The timeout feature of the phone was too short (1 minute) and a recommendation was noted that this should be extended. It was also noted that the Back and Home buttons should be enlarged some more. The Sync Online button was renamed Share. Lastly, when performing certain tasks like posting questions to the group and replying to questions posted, some participants found these features engaging.

\section{User Study 2 - Field Trials}

The second study reported in this paper focused on the actual system usage and level of collaboration afforded by this type of system. Forty participants volunteered consisting of 25 women and 15 men. There were 18 Indo-Trinidadians, 5 Afro-Trinidadians (of African ancestry) and 2 of mixed ancestry. The average age of the participants were 59.56 years (S.D. 8.2 years) and the familiarity with mobile phone was the same as before the majority of participants owned a cell phone and the primarily used it for making calls.

A half-day training session took place where the features of the application were demonstrated and 
participants were asked to practise specified tasks. Each participant was also asked to complete a Diabetes Knowledge Questionnaire before the trials started.

For this study a double-blind experiment design was utilized where each participant was assigned a unique Participant Id Code (PIC) and placed randomly in either the control or intervention group. The control group had 20 participants who received DSMS through their primary diabetes care in a clinical setting or community setting. The intervention group had 20 participants who received DSMS through the mobile-based collaborative disease management system designed by researchers at the university. After the first week of usage, the intervention group were asked to report in any problems/challenges face. These were recorded and resolved where possible.

The results presented in this paper form part of a preliminary analysis of the system usage logs after the first three months of the user trials. The aim was to investigate the frequency of usage of the different mobile application features and to identify the types of collaboration that were emerging.

\subsection{System Usage}

To investigate system usage, a logger component was designed into the application which captured defined events (each with a unique id), their timestamp (when each occurred) and optional metadata associated with the event. An algorithm was used to analyse the logs created by each user and this section presents the aggregated results of this analysis.

The most utilized feature was Trivia feature $(32 \%$ of all event entries logged were associated with this feature) followed by Discussions $(8 \%)$ and Group readings $(5.5 \%)$. At the start of every week, a new batch of questions was downloaded on the application and there was no time limit imposed on the number of times the questions can be attempted. Each successful and unsuccessful attempt was logged. The least utilized feature was the sending private Messages feature whereby a group member can contact another group member individually.

At the start of the trials there was no frequency imposed on the participants in terms of how often they were expected to use the application. In the first week the mobile application was used on average three days per week. At this time participants were not assigned to their groups as yet. The participant was expected to use this time to get familiar with the application. At the end of the first week, each user was assigned to their peer group and after the first month the application usage was on average four times per week.

In terms of the group activities, the level of collaboration differed amongst groups; this is discussed in the next section. The most popular category of discussion was the Diet category. Participants shared their daily meals including health challenges faced and during the holiday time, words of motivation and reminders were exchanged to encourage everyone to stick to their health goals. The least popular category of discussion was the Self-monitoring category.

\subsection{Collaboration}

In Frey et al. [19], the authors describe five levels of collaboration and their characteristics: networking, cooperation, coordination, coalition and collaboration. Networking being the first level; characterized by loosely defined roles, little communication and all decisions being made independently. Collaboration being the fifth level; characterized by more frequent communication, mutual trust and consensus is reached on all decisions. The data collected from the Discussion feature was analysed in terms of usage and content and the results are presented below.

The level of collaboration differed amongst groups but the most common type that emerged was coordination followed by cooperation. In coordination, the group members shared information and resources on their selfmanagement routines. They established defined roles based on the nature of the discussion taking place. There were the information seeker(s), information $\operatorname{provider}(s)$ and the mediator( $(s)$. It was noted that some roles remained the same throughout discussions. There was also frequent communication, in some cases as often as every day. Lastly there were some cases of shared decision making. For example, when a group member posed a question, more than one member would reply with a solution and some form of consensus was reached. In cooperation, the discussion feature was mainly used for providing information and it was characterized by a more formal communication structure. The discussions did not occur as often as the coordination group and all decisions were made independently and in these cases no decision was made via usage of the application.

\section{Discussion and Future Work}

The framework presented in this paper is intended to extend the remote model of peer support, to include the use of mobile technologies that will make disease management initiatives more available and accessible to the wider population. The Mobile DSMS system demonstrates how mobile devices can be used in a peer support setting to foster collaborative disease management.

The different components of the framework influenced the design and development of the application. The CDM mobile users in this context are the persons living with diabetes whose main objective is to maintain healthy lifestyles through good self-management practices. The mobile phone and the physiological meters are the main interfaces to the patient. The system is designed to ensure that input and output functions are aided given the 
constraints of the phone and the specific needs of the target group.

To establish the support context, peers are assigned to group's automatically using a custom group formation algorithm that takes into account patient specific data such as length of time with disease, current stage of illness, number of exiting complications etc. It also considers the criteria for effective group support. In the next version of the mobile application, peers will have the option to change groups as their health goals change.

For the data management and sharing, all patient data is stored on the phone and web services are used to coordinate the peer services, for example, the sending of messages and sharing of ODLs. Since ODLs are shared within each group, peers can see where each member of the group stands with respect to their health goals. This is intended to foster a form of compliance since the peer's result will be missing if not entered and competition with everyone striving to do better. This forms part of the socio-economic aspect of the system. To ensure the security and privacy of the health data, all data is encrypted before storage and transfer operations. Peers will only be able to view information generated by their own group and each peer controls what they share with their group members.

Mobile DSMS is just one instantiation used to validate the framework. Researchers can reuse the framework in any context that requires collaborative disease management. For example, a group of asthma patients sharing information on living and managing their condition, a group of expecting mothers dealing with prenatal issues or a group of children sharing information on child diseases and good nutritional habits.

The results of the first study presented indicate that the patients are willing to use mobile technology as a platform for collaborative disease management and this is just the beginning of what is needed to design an application that is suitable for the target population. Changes were made to the design of the application based on user feedback.

The updated version of the application was used in the second study. At the end of three months, the results are promising. The majority of participants are using the application regularly and the Trivia feature is the most popular followed by the group-related features. Collaboration within the groups takes place in the forms of coordination and cooperation.

At the end of the six months, the main outcomes to be measured are: participant's knowledge of diabetes, participant's problem solving ability as it related to the care of diabetes, quality of life, health locus of control, their health status (HbA1c), and their satisfaction with the peer group. It is expected that CDM will increase the access to and availability of DSMS. This will in turn result in improved self-management practices among the patient population, improved quality of life, reduce health care costs and reduced debilitating effects of the disease on individuals and economies.

\section{Conclusion}

Diabetes and other CNCDs are increasingly having a negative impact on individuals around the world as well as on economies due to costs associated with the diseases. However, these diseases are lifestyle diseases and can be controlled by DSMS. Systems that provide DSMS in developing countries are often not available and ineffective at best. Moreover, CNCDs require a different model of which current health care systems fall short. A more interactive and personalized approach is necessary at the primary health care level that increases the availability and accessibility to health care support.

This research contributes to the peer-support remote model. We presented a framework for collaborative disease management using mobile technologies. We then demonstrated, through the design of the Mobile DSMS system, how mobile technologies can enable users with similar disease management interests to virtually gather and share experiences, ask questions and provide support and problem-solve remotely through the use of mobile devices.

In order for initiatives like this to be successful, we must leverage the existing infrastructure and knowledge available in the target population and ensure that the initiative is sustainable in the long term. CDM using mobile technologies is an ideal technology-based candidate especially in developing countries where other ICT infrastructures are substandard. The Mobile DSMS system is one instantiation of this framework. It allows persons living with Type 2 diabetes to share health related information within a group setting using mobile phones. The assumption here is that through this type of informal learning and peer support, the patient's self-management behaviour will improve. The preliminary results indicate that persons are willing to use the system on a regular basis and the educational and group based activities afforded by the system were popular within the sample group. The system affords collaboration by the sharing of resources and organisation of decision making activities.

More one on one follow up will take place with the participants to better explain the recorded logs and to measure user satisfaction with the system. Even though the sample size was adequate to answer the research questions, a larger sample size and longer trial duration are required to measure health related outcomes.

\section{Acknowledgements.}

This research was supported by a grant from the Graduate Student Research Fund at the University of the West Indies, St. Augustine campus. This study was made possible due to permission granted by the University of the West Indies Ethics Committee. We wish also to thank the Diabetes Association of Trinidad and Tobago for their continued support with the project. 


\section{References}

[1] World Health Organization (WHO). Diabetes Fact Sheet $\mathrm{N}^{\circ} 172$. Updated October 2002. Retrieved from WHO website: http://www.who.int/mediacentre/factsheets/ fs172/en/index.html.

[2] Diabetes AtLAS. Retrieved from Diabetes Atlas Website: http://www.diabetesatlas.org/. Last visited 2014 June.

[3] NARACE, J. Partners' Forum for Action on Chronic Diseases in Trinidad and Tobago, 2010 March 10, Port of-Spain, Trinidad. Retrieved from Healthy Caribbean Coalition website:

http://www.healthycaribbean.org/news/110310.html.

[4] SEN, A. (1999) Development as Freedom. (Oxford: Oxford University Press).

[5] Sultan, S. AND Mohan, P. (2009). myDR: Improving the Self-Care Process for Caribbean Patients with Diabetes through Mobile Learning. In the International Journal of Education and Development using Information and Communication Technologies 5(4).

[6] Sultan S., Mohan P. and Sultan N. (2009, June). Managing Change: Experiences from a New e-Health Initiative for Patients with Diabetes and Cardiovascular Disease. Proceedings of 1st IEEE International WoWMoM Workshop on Interdisciplinary Research on E-Health Services and Systems, Kos, Greece, conference date.

[7] Heisler M. (2006). Building Peer Support Programs to Manage Chronic Disease: Seven Models for Success. California Health Care Foundation. Retrieved from The California Academy of Family Physicians website: http://www.familydocs.org/files/Building\%20Peer\%20Sup port\%20Programs\%20CHCF.pdf/.

[8] Von Korff M., Gruman J., Schaefer J., Curry SJ., Wagner EH. (1997). Collaborative management of chronic illness. Annals Internal Medicine 127(12):1097-102.

[9] Holthe H. (2006). The Integration of Peer Competence in Public E-Health Information resources. Norwegian Centre for Telemedice Project Reports: 05.

[10] Frost JH., Massagli MP. (2008). Social Uses of Personal Health Information Within PatientsLikeMe, an Online Patient Community: What Can Happen When Patients Have Access to One Another's Data. Journal of Medical Internet Research 10(3): 15.

[11] CureTogether website. Available at: http://curetogether.com/. Last visited 2010 June.

[12] Demiris, G., Finkelstein, S.M., And Speedie, S.M. Considerations for the design of a Web-based clinical monitoring and educational system for elderly patients. Journal of American Medical Informatics Association 8: 468-472

[13] SieK K.A., Ross S.E., Khan D., Haverhals L.M., Cali S.R., Meyers J. Colorado Care Tablet: the design of an interoperable Personal Health Application to help older adults with multimorbidity manage their medications. Journal of Biomedical Informatics 43(5):S22-6.

[14] Connelly, K., A. Faber, Y. Rogers, K. A. SieK, And T. Toscos. (2006). Mobile Applications that Empower People to Monitor their Personal Health. In Journal of Elektotechnik and Informationstechnik 123(4), pp.124-128

[15] PATRICK K, RAAB F, ADAMS MA, Dillon L, ZABINSKi M, Rock CL, GRISWOLd WG, NORMAN GJ. (2009) A Text Message-Based Intervention for Weight Loss: Randomized Controlled Trial. Journal of Medical Internet Research 11(1):e1.
[16] Chomutare T., Tatara N., Arsand E. AND Hartvigsen G. Designing a diabetes mobile application with social network support. Studies in Health Technology and Informatics 188:58-64.

[17] Arsand E., Tatara N., Østengen G., Hartvigsen G. Mobile phone-based self-management tools for type 2 diabetes: the few touch application. Journal of Diabetes Science and Technology 4(2): 328-36.

[18] Sultan, S. And Mohan, P. (2012). Designing a peerfacilitated self-management mobile application: A usercentred approach. In Proceedings of PervasiveHealth 2012, the 6th International Conference on Pervasive Computing Technologies for Healthcare, San Diego, CA, 21-24 May 2012, pp.228-231.

[19] Frey, B., Lohmeier, J., Lee, S. And Tollefson, N. Measuring Collaboration Among Grant Partners. American Journal of Evaluation 27; 383. 